\title{
A Ranking Algorithm for News Data Streams
}

\author{
Bhavana Mahour, \\ Department of C.S.E. \& IT \\ MITS, Gwalior (M.P.),India
}

\author{
Akhilesh Tiwari \\ Depaartment Of C.S.E.\&IT \\ MITS,Gwalior(M.P),India
}

\begin{abstract}
With the advent of internet, print media such as newspapers and magazines have been moving themselves to websites providing news on the go. Also some people prefer news on the net more as pictures along with video is available in it, also we can search a particular topic. In this paper we suggest a ranking algorithm that exploits the dependency between the rank of news article, topic and source by the help of a virtual graph model. We also add the imitating or copying of new articles to revise the ranking of a source or article. Our complexity is linear and for starters we have just added the national news channels in our algorithm. To validate our algorithm we use huge manual data collecting news from national news websites.
\end{abstract}

\section{Keywords}

News Ranking, articles, source, topic, Time aware algorithm

\section{INTRODUCTION}

With terabytes of news adding every day on the internet and a plethora of news sources available including international news channels such as BBC, CNN and along with national news channels such as AAJTAK, ZEE NEWS moving themselves to world of internet. For the convenience of users a number of search engines are available on the internet such as Google, Yahoo news on the net but we consider there ranking criteria is based on commercial use as to who will pay more will be on the top.

"The Internet complements television for news coverage as it provides a different perspective and greater depth of information - statistics, pictures, interactive maps, streaming video, and analyst comments," said Peter Steyn of Nielsen/Netrating.

In past few years, more and more people have been using the internet not just for entertainment but also reading news on the web. According to a recent survey made by Bielsen netRatings [7], the number of people browses news on the web to know what's happening around the world. It has become part of our daily life. User easily accesses the news information on the web. There are more commercial news search engine available such as Google News [8], Yahoo News [9] NewsInEssence[10] and newsbot [12] providing news information according to their will. Google News search provide the information, aggregates headlines from news sources worldwide, group's similar stories together and displays them according to user's personalized internets. Yahoo News [3] search engine provide the news information with similar services and another important news search engine NewzsInEssence[2] which provide the news information, clustering and summarizes similar news articles. In Liu et al. proposed an algorithm for ranking news related to some query submitted at a specified timestamp [5].

In the research area, a lot of work has been done in the field of web page ranking. Page ranking method are based on the linking structure of web graph such as Page Rank [6], HITS [8] SALSA [7] and Berberichet al. [9] many techniques are used to the page ranking algorithm.
Introduce a time aware ranking method [2]. Time aware rank method differs from Page Rank method [3]. it considers web pages modified time and activity during ranking. The time aware ranking [6]. Provide fresh information on news articles thus we have to develop new Ranking methods to solve the problem. In an online news ranking method is propose to news source and related articles, copy right articles source to another source,

In this paper, the problem of ranking algorithm for news source, articles ,topic and copyright articles source to another source. And also we propose a graph model to formalize the relationship between news sources, articles and source to another source. Our ranking algorithm is based on this model and reflects the mutual reinforcement between news source, articles and source to another source.

\section{PROPOSED METHODS}

\subsection{News Properties}

If we have to rank a news article, it is all the way different from ranking web pages since it contains very less or no HTML links which can be exploited as in PageRank[15] hence we have to determine the rank based on the virtual linking between topics, articles and sources. The most important properties are discussed below.

\section{Ranking for News posting and News sources}

We have to devise an algorithm that separately calculates the rank of source and articles as well as also topics which are most read about.

\section{Time awareness}

The importance of a news item changes over time for the reason that we are dealing with a stream of information in which a fresh news story can be considered more important than an old one. Thinking of news as a stream of data, the rank of news will decrease as the time elapses.

\section{Important News articles are clustered}

It is probable that an important news article $\mathrm{n}$ will be (partially) replicated by many sources, considering for example, a news article $n$ originated from a press agency. A measure of its importance can also be gauged by the number of different on-line newspapers which either replicate or extract sections of text from $\mathrm{n}$. The phenomenon of using stories released by other sources is common in the context of Web journals and, as far as the news engine is concerned, this means that the (weighted) size of the cluster formed around $\mathrm{n}$ can be deemed a measure of its importance.

4. Mutual Reinforcement between articles and Sources A lot of articles are generated daily and we decide the rank of the article of the same heading coming from two different sources, then the source having more credibility or have the reputation of giving good news such as "Zee News" will be 
given more importance than an article by a local or state level source.

\section{Source Authority}

Different news web sites have different authorities. Suppose two nearly identical news articles, one is published by ZeeNews, the other is from an unknown web site. Since ZeeNews is a famous and credible news website, we would expect the news from ZeeNews having higher rank. Also news topics may overlap with each other.

\section{Mutual Reinforcement of imitating Sources}

We take into consideration the sources which copies the whole article or part of the article in its own website. We use this to calculate the rank of the parent source(from which the article is originating) by increasing its rank depending on the amount of its copying and also since the others are copying it; this reduces the credibility of the other sources and thus reducing their rank by considering the amount of imitation by them.

\subsection{VIRTUAL GRAPH MODEL}

Although there is little explicit HTML links between news web pages, we can create virtual link between source, topic, articles and copied articles. We define a virtual undirected link graph $\mathrm{G}$ $=(\mathrm{V}, \mathrm{E})$, where $\mathrm{V}=\mathrm{S} \cup \mathrm{C} \cup \mathrm{N} \cup \mathrm{N}^{\prime}$. Herein, $(\mathrm{S}$ stand for the set of source, $\mathrm{N}$ stands for the set of articles; $\mathrm{C}$ stand for the topics; and N' stand for the articles which have been copied). The virtual set $\mathrm{E}$ contains four disjoint partition; E1 E4. E1 represent the news creation process. E2 represent the similarity between topic and articles (news stream) ; when articles ai belongs to topic $t_{j}$ there's an undirected edge between $a_{i}$ and $t_{j}$. E3 it is a relation between topic and source in which an articles $a_{j}$ is copied by source $s_{j}$ this nodes indicates our copy right property also this will reduce the ranking of source $\mathrm{s}_{\mathrm{j}}$. E4 it is the fresh article published by source $s_{j}$ which is copying other articles from other source.

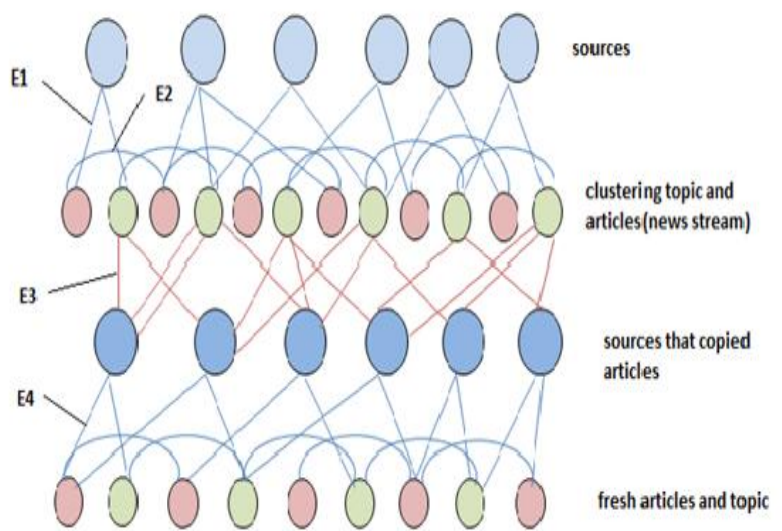

Fig1. News virtual link graph

We use weighted adjacency matrix to express the graph and denote it as
Where $\mathrm{P}, \mathrm{Q}, \mathrm{S}$ refer to edges from source to topic source to article and topic to articles respectively , $\mathrm{R}$ is refer to edges from copy right articles and $\Sigma$ is the similarity matrix. We can use a sub matrix at the upper-left corner of A instead of $\mathrm{O}$.

\subsection{STREAM CLUSTRING}

We could use a clustering algorithm to cluster similar articles from topic.

\subsubsection{Similarity Computation}

We have used a manual approach for similarity computation. We have given our articles/topics to some individuals which give us a value of similarity on a scale of $0-10$ converted into decimal number.

\subsubsection{Clustering}

Our proposed algorithm uses bottom up approach and expect our cluster to be highly coupled. Our topic cluster $T$ has a constant parameter $T H(0 \leq T H \leq 1)$ for similarity threshold between topic and article also we merge two articles in a cluster by using threshold parameter $H(0 \leq H \leq 1)$ by comparing the contents of the articles as well as the time difference between them $t$ and defining them into a topic cluster.

\section{Proposed Algorithm}

Input: $\mathrm{T}-\mathrm{a}$ set of individual cluster of topics $\mathrm{T}=$ $\left\{\mathrm{T}_{1}, \mathrm{~T}_{2}, \mathrm{~T}_{3} \ldots \ldots \mathrm{T}_{\mathrm{n}}\right\}$, incoming articles $\mathrm{a}_{\mathrm{j}}$, articles threshold $\mathrm{H}$, topic threshold TH,Time difference between articles $\mathrm{t}$

1. For all incoming articles $a_{j} \in A$,

$A=\left\{a_{1}, a_{2}, a_{3}, a_{n}\right\}$ set of articles.

2. For each $T_{i}, \operatorname{check} \operatorname{sim}\left(T_{i}, a_{j}\right)>T H \quad\{i=1$ to $n\}$

3. For each $a_{j} \in A$ initialize cluster $\}$

4. Choose $\left(a_{i}, a_{j}\right) \in A X$ A with $\max \operatorname{sim}\left(a_{i}, a_{j}\right)$

5. Check $\operatorname{sim}\left(\mathrm{a}_{\mathrm{i}}, \mathrm{a}_{\mathrm{j}}, \mathrm{t}\right)>\mathrm{H}$

6. Set merge $==1$

7. $a_{k}=a_{i} \cup a_{j}$

8. $\quad$ add $\mathrm{a}_{\mathrm{k}}$ to $\mathrm{T}_{\mathrm{i}}$

9. else return

10. repeat till $\mathrm{T}_{\mathrm{n}}$

11. end

\subsection{Matrix Expressing}

The naïve approach is that a news sources has a rank proportional to the number of pieces of news it generates and conversely, that a news articles should rank high if there are many other news stories close to it formally, denoting by $r=\left[r_{S}\right.$ $\mathrm{r}_{\mathrm{SC}}, \mathrm{r}_{\mathrm{N}]}^{\mathrm{T}}$ the vector of news source, copied news source and rank of news stream, we can compute them as

$$
\mathrm{r}=\mathrm{Ar}
$$

$r=\left[r_{S}, r_{S C}, r_{n}{ }^{T}\right.$ where $r_{S}$ represents vector from news source, $r_{S C}$ represents vector copied news source or the news source which 
copied other articles and $r_{n}$ is the vector representing rank of news stream.

$$
\begin{gathered}
\mathrm{r}_{\mathrm{S}}=\mathrm{Pr}_{\mathrm{n}}+\mathrm{Qr}_{\mathrm{SC}} \\
\mathrm{r}_{\mathrm{SC}=\mathrm{p}^{\mathrm{Trs}}{ }_{\mathrm{Rrn}}+\mathrm{Rrn}} \\
\mathrm{r}_{\mathrm{n}=}=\mathrm{Q}^{\mathrm{T}} \mathrm{r}_{\mathrm{s}}+\mathrm{S}^{\mathrm{T}} \mathrm{r}_{\mathrm{n}+\sum} \mathrm{rn}
\end{gathered}
$$

the ranks of sources are decided by the rank of related topic and published articles; the rank of topics are decided by the ranks of related sources, similar topics and articles contained; the rank of articles are decided by the ranks of the sources publish them, the topics belong to and other similar articles

\section{TIME-AWARE RAKING ALGORITHM}

To deal with a news data stream we have to design time aware mechanisms, which do not use fixed time observation window over the flow of information. They key idea is that the importance of a piece of news is strictly related to the time of his emission. Hence, we model this phenomenon in traducing a parameter $\alpha$ which accounts for the decay of "freshness" of the news story. This $\alpha$ depends on the category to which the news articles and topic belongs. For instance, it is usually a good idea to consider sport news.

We denote the rank of some source $s$ as $R(s, t)$ at time $t$; the rank of some topic $c$ as $R(c, t)$ at time $t$ the rank of some articles $n$ as $R(n, t)$ at time t. we use $S\left(n_{i}\right)=s_{j}$ to represent that articles $\mathrm{n}_{\mathrm{i}}$ is published by source $s_{j}$ an use $C\left(n_{i}\right)=c_{j}$ to show that articles $n_{i}$ belongs to topic $c_{j}$

\section{a. Decay Rule}

We adopt the following the exponential decay rule for the rank of $n_{i}$ which has been released at time $t_{i}$.

$$
R\left(n_{i}, t\right)=e^{-\alpha(t-t i)} R\left(n_{i}, t_{i}\right), \quad t>t_{i}
$$

The value $\alpha$ is obtained from the half-life decay time $\rho$ that is the time required by the rank to have its value, with the relation

$$
\mathrm{e}^{-\alpha \rho}=\frac{1}{2}
$$

In the following, we will specify the parameter $\rho$, expressed in hours, instead of $\alpha$. Besides, we discuss how to obtain the formulation of an effective algorithm for ranking news articles, topic and sources.

The constant parameter $\alpha$ in (1) decides the rate new a rank decay: it can be computed by (2) given the half-life decay time $\rho$ which stands for the time in which the rank halves its value. We can give different $\rho$ to different kinds of articles, thus have a control over their decay process,e.g. we can assign larger $\rho$ to policy related news than sports news since the policy news decays slower. Similarly, we decay the rank of some topic $c_{i}$ according to (3)

$$
R\left(c_{i t}\right)=e^{-\alpha(t-t i)} R\left(c_{i}, t_{i}\right), \quad t>t_{i}
$$

Now we will consider the decaying of rank as time goes by. Let $\rho$ decay time, $\beta$ jump probability, $\gamma_{\mathrm{ij}}=1$ (copyright articles), $\gamma_{\mathrm{ij}}=$ 0 (unique no copyright articles). Let $\mathrm{s}$ source, $\mathrm{c}$ topic, $\mathrm{n}$ articles $\mathrm{s}_{\text {num }}\left(\mathrm{c}_{\mathrm{i}}, \mathrm{S}_{\mathrm{k}}\right)$ be the number of articles published by $\mathrm{s}_{\mathrm{k}}$ in topic $\mathrm{c}_{\mathrm{i}}$ num $\left(c_{j}\right)$ number of articles in topic $c_{j}$.

\subsection{Algorithm TA1}

$$
\begin{aligned}
& \text { Rank of source }\left(\mathbf{s}_{\mathbf{k}}, \mathbf{t}\right) \sum_{s\left(n_{i}\right)=s_{k}} e^{-\alpha\left(t-t_{i}\right)} R\left(n_{i}, t_{i}\right)^{\beta}+ \\
& \sum_{t i>t j} e^{-\alpha\left(t_{i}-t_{j}\right)} \gamma_{i j} \mathrm{R}\left(n_{j}, t_{j}\right)^{\beta}- \\
& \sum_{t_{k<t_{i}} e^{-\alpha\left(t_{k}-t_{i}\right)}} \quad \gamma_{i j} \mathrm{R}\left(n_{k}, t_{k}\right)^{\beta}
\end{aligned}
$$

Similarly we can deduce the rank of topics:

\subsection{Algorithm TA2}

\section{Rank of topic $\left(\mathbf{c}_{\mathbf{k}}, \mathbf{t}\right)$}

$$
\begin{aligned}
& =\sum_{c\left(n_{i}\right)=c_{k}} e^{-\alpha\left(t-t_{i}\right)} R\left(n_{i}, t_{i}\right)^{\beta} \operatorname{sim}\left(n_{i}, c_{k}\right) \\
& +\sum_{\operatorname{snum}\left(c_{k}, s_{m}\right) \neq 0} R\left(s_{m}, t\right)^{\beta} \frac{\operatorname{snum}\left(c_{k}, s\right)}{\operatorname{num}\left(c_{k}\right)}+\sum_{t_{i>t_{j}}} e^{-\alpha\left(t_{i}-t_{j}\right)} \gamma_{i j} \mathrm{R} \\
& \left(n_{j}, t_{j}\right)^{\beta} \operatorname{sim}\left(n_{j}, c_{k}\right) \ldots \ldots . . .(5)
\end{aligned}
$$

\subsection{Algorithm TA3}

Rank of articles $\left(\mathbf{n}_{\mathbf{k},}, \mathrm{t}\right)=\mathrm{R}\left(\mathrm{s}\left(n_{K}\right), t\right)^{\beta}+e^{-\alpha\left(t-t_{i}\right)}$

$$
\begin{aligned}
& \frac{1}{n u m c_{j}} \mathrm{R}\left(\mathrm{c}\left(n_{k}\right), t_{i}\right)^{\beta} \operatorname{sim}\left(n_{i} c\left(n_{k}\right)\right) \\
& \left.+\sum_{t_{i}<t_{j}} e^{-\alpha\left(t_{i}\right.}-t_{j}\right) \gamma_{i j} \mathrm{R}\left(n_{j}, t_{j}\right)^{\beta} \operatorname{sim}\left(n_{i}, n_{j}\right)
\end{aligned}
$$

\section{EXPERIMENT}

Our experiment is based on sub set of data collected from websites of national news Channels such as AAJTAK, Zee News, India TV and NDTV for a period of 15 days. The code is written in PHP and the ranking of about 500 news pieces of information. During our experiments, we set the half-life decay time $\rho$ to be 24 hour, random jump probability $\beta$ to be 0.2 , and the clustering similarity threshold $\mathrm{H}$ to be 0.7 and topic threshold TH 0.3

Table 1. top 5 rank topics

\begin{tabular}{|l|l|l|}
\hline \multicolumn{1}{|c|}{ Rank } & \multicolumn{1}{|c|}{ Topic } & \multicolumn{1}{c|}{ Source } \\
\hline 12.26 & Delhi election 2013 & Zee TV \\
\hline 19 & Terrorist Attack & STAR TV \\
\hline 28.66 & Sport news & Zee TV \\
\hline 14.4 & Natural disaster & NDTV \\
\hline 44.49 & Politics Of Religion & $\begin{array}{l}\text { INDIA } \\
\text { TV }\end{array}$ \\
& & \\
\hline
\end{tabular}


Table 2. top 5 rank articles

\begin{tabular}{|c|l|}
\hline Rank & \multicolumn{1}{|c|}{ Articles } \\
\hline 134.8 & Delhi poll: records 67\% voters turnout \\
\hline 40.66 & Blast in Delhi NCR kills 7 and several injured \\
\hline 209.9 & $\begin{array}{l}\text { 2 CRPF personnel killed in terrorist attack in Jammu } \\
\text { and Kashmir }\end{array}$ \\
\hline 66.9 & Uttrakhand floods \\
\hline 96.89 & 2013 India and south Africa test series \\
\hline
\end{tabular}

Table 3.top 5 rank articles

\begin{tabular}{|c|c|c|}
\hline Rank & $\begin{array}{c}\text { No. Of Articles Copied By Other } \\
\text { Sources }\end{array}$ & Sources \\
\hline 3.94 & 14 & NDTV \\
\hline 4.77 & 9 & ZEE TV \\
\hline 0.25 & 7 & INDIA \\
& & TV \\
\hline 9.16 & 7 & STAR TV \\
\hline 2.74 & 5 & AAJ TAK \\
\hline
\end{tabular}

To find the precision of our algorithm we used number of clicks done by users for a particular topic/source/articles consisting of a group of 10 people and we match the results with our calculated ranking by the formula $P_{s}=n(S) / K_{s}$ x 100 where $n(S)$ is the total number of clicks for sources $S$ and $K s$ is our ranking judged to be important by our algorithm. Similarly ranks of topics and articles can be calculated. By our experiments we find that the precision of topics and articles decreases as after time a lot of articles can be seen for a particular topic/news are available so the user is not restricted to accessing a particular article only.

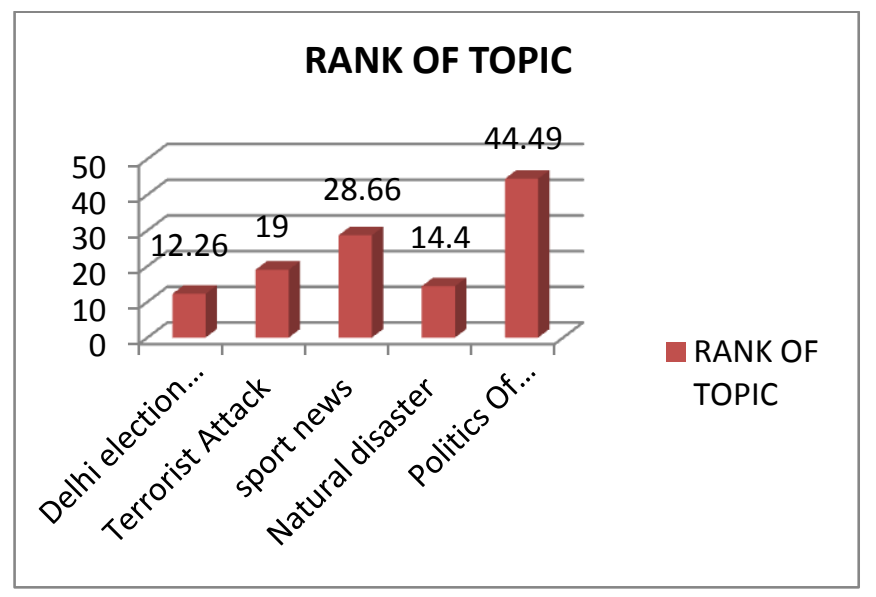

Figure 5.1 Ranks of Topic

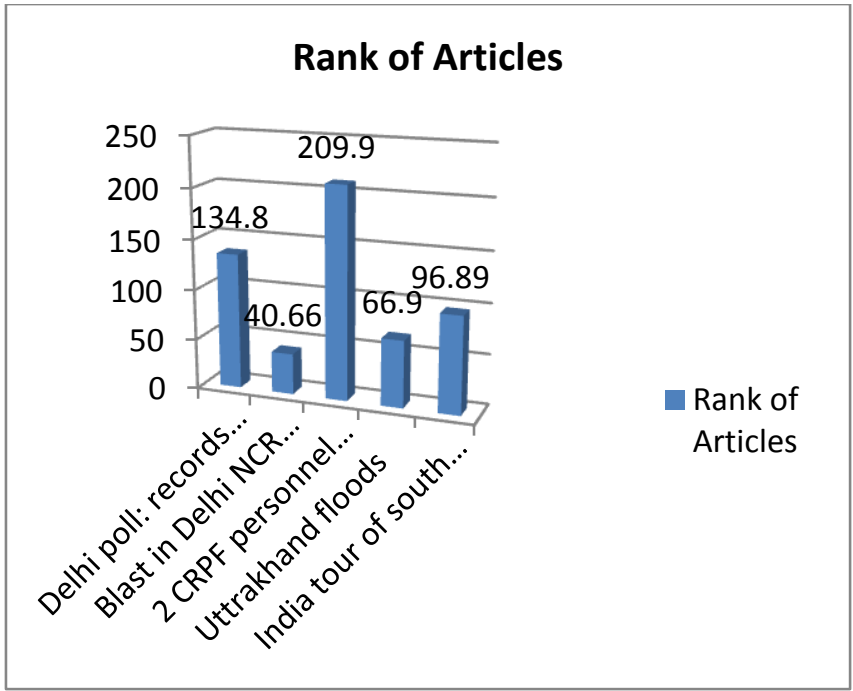

Figure 5.2 Ranks of Articles

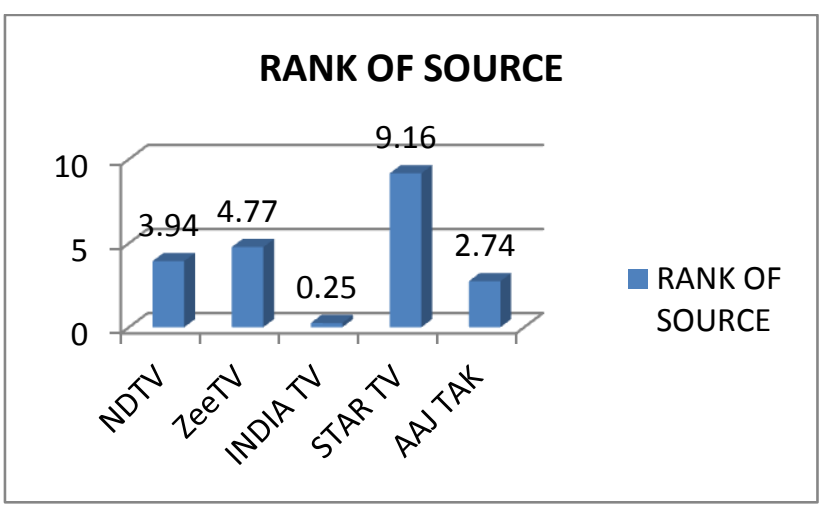

Figure 5.3 Ranks of Source 


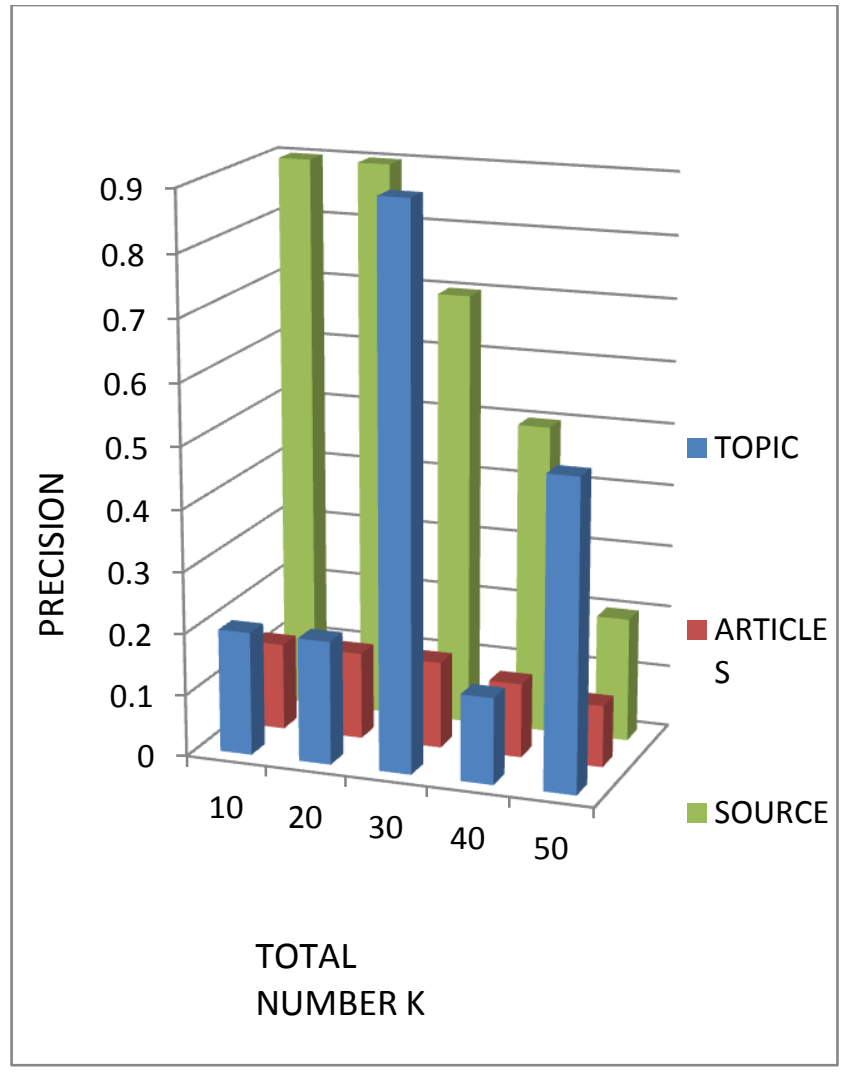

Figure 5.4 Ranks of Precision

\section{CONCLUSION AND FUTURE WORK}

Our algorithm calculates the ranking of sources, topics and articles altogether the news stream. The data collected from national news channels for a period of 15 days shows encouraging results and the order is also increasing linearly .Future work for our algorithm is the personalization of news in which the data of every user logged also need to be taken and then news is displayed. Similarly it can be used to rank research papers and also popularity of a particular page in social networking sites such as Facebook where people search for related pages.

\section{REFERENCES}

[1] Xi Mao, Wein Chen, "A Method For Ranking News Sources, Topic And Articles," In proceeding of the $2^{\text {nd }}$ International Conference On Computer Engineering And Technology (IEEE), 2010 vol.4.

[2] Gianna M. Del Corso, Antonio Gulli, Francesco Romani, "Ranking A Stream Of News," In proceeding of The
International World Wide Web Conference Committee (IW3C2) 2005.

[3] Dilip Kumar Sharma, A. K. Sharma, "A Comparative Analysis Of Web Page Ranking Algorithm.” In proceeding of the International Journal On Computer Science And Engineering 2010 vol.02.

[4] L. Page, S. Brin, R. Motwani,And T. Winograd. The Pagerank Citation Ranking: Bringing Order To The Web. Technical Report, Standford Digital Library Technologies Project, 1998.

[5] X.F. Liu, C.B. Chen and Y.S. Liu, "Algorithm For Ranking News," In Proceeding Of The Third International Conference On Semantics, Knowledge And Grid, 2007.

[6] K. Berberich, M. Vazirgianins and G. Weikum," T-Rank: time aware authority ranking," In Proceeding Of The WAW 2004.

[7] R. Lempel and S. Moran, :The Stochastic Approach For Link-Structure Analysis(SALSA) And The TKC Effect, "In proceeding of the $9^{\text {th }}$ International World Wide Web Conference, 2000.

[8] J. Kleinberg, "authoritative sources in a hyper-linked environment, "In proceeding of the $9^{\text {th }}$ ACM-SIAM symposium on discrete algorithm 1998

[9] [17] R. Kosala And H. Blockeel, "Web Mining Research: A Survey", In ACM SIGKDD Explorations, 2(1), PP.1-15, 2000.

[10] Harmunish Taneja, Richa Gupta "Web Information Retrieval Using Query Independent Page Rank Algorithm". 2010

[11] Xin Li, Bing Liu, Philip Yu "Time Sensitive Ranking with Application to Publication Search". 2008

[12]Xiang Lian And Lei Chen, "Ranked Query Processing In Uncertain Databases", In IEEE KDE, Vol. 22, No. 3, March 2010.

[13] Shiguang Ju, Zheng Wang, Xia Lv, "Improvement of Page Ranking Algorithm Based on Timestamp and Link". 2008

[14] Robin Sharma, Ankita Kandpa, Priyanka Bhakuni, Rashmi Chauhan, R.H. Goudar "Web Page Indexing Through Page Ranking For Effective Semantic Search" 2012.

[15] Ling Wan, Si-Xue Bai "An Improvement Of Pagerank Algorithm Based On The Time-Activity-Curve". 